\title{
A Study on Inherent Mechanism of Collaborative Innovation to Promote the Reform of Science and Technology Research Organization in Modern University
}

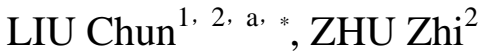 \\ 1. Wuhan Technology and Business University, Department of Management, Wuhan 430065
}

2. Hubei Collaborative Innovation Center for Modern Logistics and Commerce, Wuhan 430065

a. Lc576@126.com

\begin{abstract}
Collaborative innovation is a new paradigm of modern university science and technology research, which is based on the needs of social development. It is not only the inevitable choice for the university to improve the innovation ability and the efficiency of scientific research, but also the endogenous drives for the innovation of modern university from division to integration, and from decentralization to integration. Based on science and technology research idea of knowledge production and dissemination, the shackles of bureaucratic culture and single defense preparations, the science and technology research organization structure of modern university hinders the promotion of collaborative innovation. Therefore, under the guidance of collaborative innovation concept, the existing university science and technology research organization must be reformed to meet the requirements of internal and external coordination. From the perspective of collaborative innovation, this paper analyze organization, culture, resources and other related factors of modern university science and technology research reform, and construct a theoretical model of science and technology research organization reform, in order to promote the science and technology research organization reform in modern university.
\end{abstract}

Key words: Collaborative Innovation; Modern University; Science and Technology Research Organization

\section{Introduction}

Collaborative innovation is a new model of innovation and development. In recent years, its development has shown the characteristics of diversification. And in the course of its development, it gradually increased the level of ideas, gradually deepened the span, but also collaborative innovation crossed the various disciplines. However, in the process of its development, it has been constrained by the organizational mechanism, the interests of various disciplines, and ideas. Collaborative innovation is difficult to collaboration, discipline is the foundation of university internal collaboration. discipline as a platform converge key elements of collaborative innovation - personnel, structure and goals, these elements of contact between each other and interact with each other, are driving the transformation and innovation of modern university science and technology research 
organization.

\section{The Elements of Science and Technology Research Organization Reform}

The inevitable product of social development is the organization, and all kinds of social activities are developed on the basis of the organization as the basic unit. Scientific innovation play an important role in all organized activities, it is one of the ways for people to learn knowledge, a feasible way for human to explore the unknown. The scientific study of human beings is carried out by scientific organization. Scientific organizations combine scientific equipment, human resources and scientific research funds by their own strength. Of all the scientific organizations, the most important is the university scientific research organization, which integrates scientific research institutes, various departments, scientific research center. Based on the academic characteristics of university scientific research organization and the essence of synergy, also referring to the seven elements of enterprise organization designed by McKinney, this paper summarize the main factors of the scientific research organization for change as the organizational elements, cultural elements and resource elements, the organizational elements among them includes objectives, organization and structure.

\subsection{Organizational Elements}

The specialization and synthesis of scientific development is more and more obvious, the rise of interdisciplinary and interdisciplinary subject makes the gap between traditional disciplines gradually filled, there is a multi-level unity among different disciplines. The research paradigm of collaborative innovation makes the goal of scientific research organization high fuzziness and roughness. However, if an organization is to achieve the expected, made the achievements, it must have the goal clear and able to guide the activities of the people. Academic leaders within the scientific research organization must divide the fuzzy and uncertain scientific innovation goals into practical small targets in each stage, and in the implementation process according to the actual situation to make the adjustment, and harmonize the ultimate goal and stage goals, organizational goals and individual goals, in order to stimulate the potential of each member in scientific research organization, get a synergy of the research organizations.

Scientific research organization is a knowledge intensive organization. Researchers are typical knowledge workers. The collaborative innovation is an interdisciplinary, inter-department, cross-organizational knowledge integration activities, which requires the researchers not only have the ability of independent public relations and teamwork, but also have their academic knowledge and related discipline knowledge. The characteristics of university scientific research activities is the exploration and innovation, University researchers must have the spirit of collaborative innovation, university 
scientific research organization brought together the direction of scientific research, teaching and research equipment, and human resources, have the conditions to conduct scientific innovation and complete technical research. The integration of different disciplines in universities provides an effective way for the communication of study ideas and the exchange of information. It is a platform to cultivate interdisciplinary, comprehensive and complementary disciplines.

\subsection{Cultural Elements}

The cultural elements of scientific research organizations usually depend on the internal beliefs of the researchers and the external institutional environment of the organization. The cultural structure of scientific research organization is an important condition to realize collaborative innovation. The university to participate in collaborative innovation activities, will create a value integrated culture that covers change, in the early stage it may be a manifestation of the external system, then gradually formed a kind of inner faith, and mutual diffusion and influence each other among the university scientific research organizations, they formed a new culture after the reform2. Organizational culture can limit change, and lead to change. As the heart of the University, the cultural system of scientific research organization is the foundation of the whole university organizational culture, and determines the vitality and tension of their own reform.

Collaborative innovation of university is a large and complex scientific research activity, to make the whole organization synergy effects formed by the multi subject and object taking part in research is greater than the sum of the individual synergistic effect, and in order to strive for the realization of knowledge innovation and application, reach a consensus on the concept of collaborative innovation in scientific research, build a collective, flowing, open and integrated collaborative innovation atmosphere converging human resources, material resources, financial resources and other resources. Collaborative innovation is based on the major national needs, continue to focus on economic, environmental, health, population and other social issues, promote the sense of social responsibility and mission of university scientific research organization for the development of academic research and technology progress. The researchers attach great importance to the public interests of the society to form a strong cooperative belief, stimulate the collaborative innovation motivation of scientific research personnel, so as to build a scientific research team with highly cooperative consciousness. The scientific research organization implement change not only under the condition of scientific research resources, such as human, finance and material, but also under the cultural conditions of scientific research team. As the organization of knowledge application and innovation, scientific research team must have an innovative cultural environment suitable for the development of knowledge. Innovation culture affects team and members 
knowledge, on this platform, knowledge can vertically and horizontally communicate with each other in high frequency and deep level, these exchanges of knowledge provide the conditions for the effective application and innovation of knowledge. It is extremely important to create an innovative cultural environment conducive to the exchange of knowledge in the scientific research team.

\subsection{Resource Elements}

The resource factor is the key to the survival of the organization, and the essence of scientific research organization reform based on collaborative innovation is a process of interaction, integration and reorganization of resources. The resource elements of scientific research organization change are the technical methods and tools system used by the researchers in the process of collaborative innovation. Information, as the carrier of knowledge, has a close relationship with the circulation of knowledge. Informatization means and information resources play an extremely important role in knowledge fusion. The modern communication and network technology eliminates the spatial constraints of knowledge, so that the boundaries of the organization more broad, the sources of the knowledge more extensive.

A new type of the scientific research organization conducive to collaborative innovation is a product to break the original organizational barriers after the integration of scientific information, scientific instruments, scientific research funds, scientific research personnel and other key elements. The vitality of scientific research team relies on key disciplines, interdisciplinary, key laboratories, scientific research bases and other resources. The systematization of all kinds of innovation conditions is realized by collaborative innovation, which can ensure the smooth circulation of various innovative elements in the scientific research organization. Therefore, we must establish interdisciplinary and cross-organizational entity scientific research platform or base, change the traditional allocation mode of scientific research resources, enrich the way of knowledge communication between team members, so that knowledge can break through time and space constraints, achieve the optimization and integration of scientific and technological resources.

\section{Relationship and Models between Elements}

\subsection{Relationship}

It is not enough to produce a new type of organization, which has only three important elements: organization, culture and resources, but not for integration, interaction, play a role. Only the above three elements are connected with each other and interact with each other. to form a community, to play their respective role in the overall framework of organization, become an indispensable content organization, the new 
organizational system was formed.

The organization must have a combination of institutional environment to play a role, to regulate the relationship between the various factors or resources, coordinate the interests of different personnel in the organization, so that they have a unified goal and behavior norms. This institution must be matched with the resources in the organization, only in this way, the resources of the organization can give play to the maximum extent. Culture plays a pivotal role in all the organization resources, it is the basis of all the elements of the organization, is the representation of the organization values and ideas, so that the organization can be essentially run.

In the organizational system, the goal is their core, which leads to the connection of other elements in the organization. In turn, the connection of these elements often restricts the realization of the goal. Therefore, when collaborative innovation has become a new target of university scientific research, it is necessary for the collision, infiltration and fusion of multi disciplinary ideas and methods. The interaction of energy, material and information requires the optimization and integration of innovation resources, and innovative elements flow freely in the system, so as to achieve the effect of synergy "1+1>2" rather than the original effect of "1+1=2".

\subsection{Theoretical Model}

In the system of university scientific research organization, the key elements such as the goal, the structure, the personnel, the resources, the culture and the operation mode are all connected to each other surrounding the knowledge form of discipline. The discipline is a "resource community", which consists of three types of resources: the scholars, mainly refers to the expert group specializing in academic research and scientific research team; academic information, mainly refers to the knowledge and its carrier and information exchange level; academic material basis, which engaged in teaching and scientific research funds, equipment and facilities. In addition, the discipline also includes behavioral norms and values, namely the discipline culture3. Therefore, the foundation of university internal collaboration is discipline, whether it is the discipline construction, the scientific research or the talents cultivation, these all need to be reflected by the discipline.

Under the impetus of the innovation demand, the organizational elements, as an organic whole, carries out the exchange of information and energy in the new organizational system revolving around the discipline of collaborative innovation. Due to scientific research organizations attached to various disciplines, there are some differences among the organizational characteristics, organizational goals and organizational boundaries.

In the innovation process of scientific research organization, research organizations 
with the help of transfer, sharing, learning and digestion and absorption of the elements between personnel and disciplines, personnel and personnel, disciplines and disciplines, realize the updating of internal elements of collaborative organization, and make each element form a new structure, so as to complete the original organizational change.

In the whole process of change, resources are the key elements of the survival of the scientific research organization system, the new function and new force of scientific research organization system come into being from the integration of human resources, material resources, financial resources and information resources. The cultural elements run through the whole process of interaction, and constantly change and develop with the combination and recombination of internal and external elements of organizational system.

Therefore, the change of scientific research organization will be accompanied by the interaction of the elements of organization, resources and culture. The specific model is shown in figure 1.

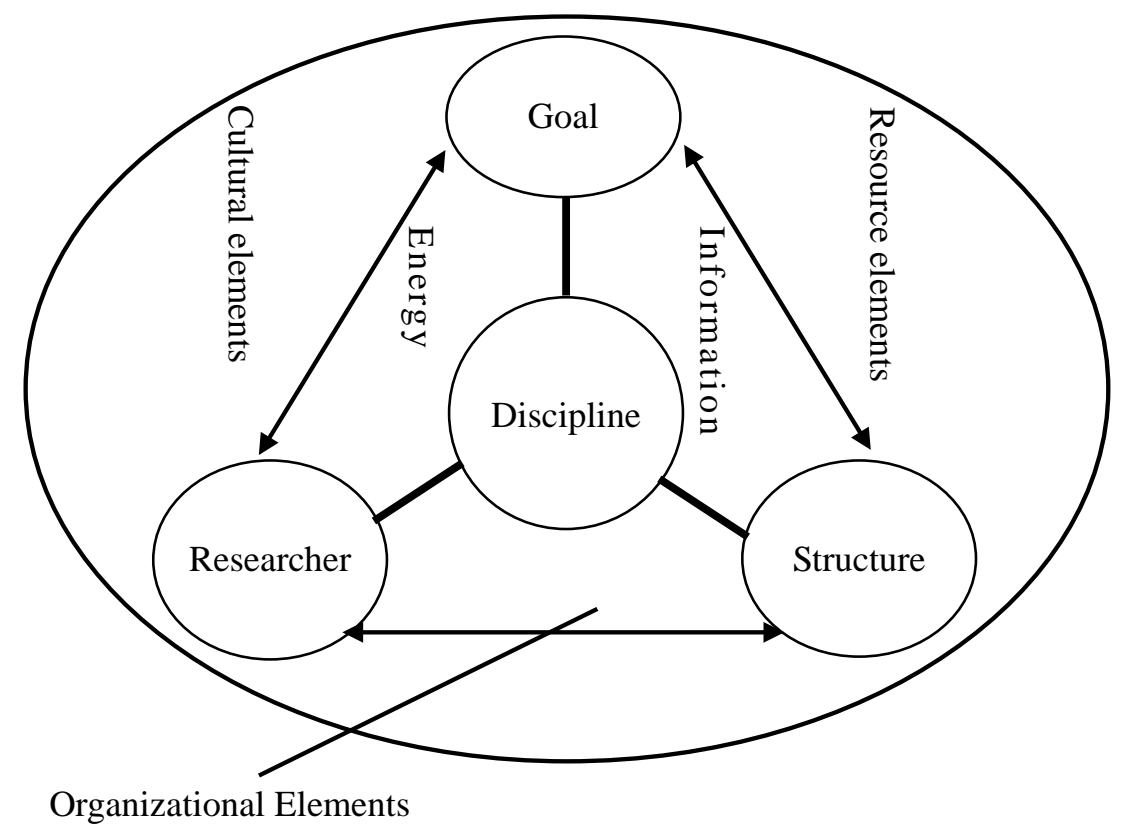

Figure 1 Theoretical model of organizational change

The establishment of modern universities is based on the organizational structure of discipline class of the College / Department, the school provides the College / Department funding and resources, the Institute plans its own recruitment, assessment and promotion. Under such a system, human, material, financial and other elements are difficult to integrate and circulate. To some extent, The self-enclosed organizational structure of University makes it difficult to carry out collaborative innovation. 
Therefore, to establish a scientific research organization to adapt to collaborative innovation within the university, must break the discipline barriers, open the boundaries between disciplines, cross the barriers of the College / Department. To form a new interdisciplinary innovation organization, make full use of synergies between the various disciplines, enhance the interaction of energy, material and information between the relevant disciplines. Finally, produce new functions and new forces on the integrity of innovative scientific research organization, rather than the simple superposition of each element function.

\section{The Realization Pattern of Scientific Research Organization Reform in Modern Universities}

According to the above analysis of the elements related to the change of the scientific research organization in the modern university, this paper constructs a theoretical model of the interaction among the organizational elements, the cultural elements and the resource elements in the change of the scientific research organization. It is found that the key elements, such as goal, structure, personnel, resources, culture and mode of operation, are related to each other in the form of subject knowledge.

It has become the focus of scientific research organization in the modern university to integrate the advantage resources, reasonably allocate the resources, optimize the allocation of scientific and technological personnel based on the advantages of multidisciplinary convergence, enhance scientific research strength, establish a scientific research organization to adapt to collaborative innovation.

This paper will analyze the change path of the scientific research organization of the modern university from three aspects: the belief, the power and the structure of the university, so as to put forward the realization way of scientific research organization reform.

\subsection{Make Clear the Goal of Change, Reform the Concept of Scientific Research Organization}

With the innovation of science and technology and the development of economy, a single research field or technical methods can not solve the social and economic problems well. Therefore, interdisciplinary research is not only the impetus of external environment, but also the endogenous demand of knowledge development. In order to promote the communication and cooperation among the subjects of scientific research activities, we must establish the scientific research idea of interdisciplinary collaborative innovation, and make it cross the double barriers of academic knowledge and academic thought.

First, academic knowledge barriers. academic knowledge for different disciplines of 
is different, each discipline has an independent and specialized expression for the objective phenomena and the law of things. The researchers from different disciplines have differences in Scientific thinking, value thinking, contingency thinking, so that they may be difficult to understand and tolerate each other behavior and value judgments, and the resulting contradictions will hinder the penetration of knowledge between disciplines.

Second, academic thinking obstacles. It is difficult for researchers of different disciplines to form the same cognitive style and sense of worth. In the multidisciplinary collaborative innovation process, they may have differences and conflicts in the method of scientific research or the selection of technical route. In addition, the lack of scientific and sound assessment system, as well as relaxed and harmonious atmosphere of scientific research, thereby affecting the entire innovation team collaboration.

\subsection{Balance the Two Type Powers, Reform the Scientific Research Organization System}

From the formal point of view, the reform of scientific research organization in modern university is the reorganization and reform of organizational structure, and in essence reallocate innovation resources and adjust academic power and administrative power. Nowadays, from the academic point of view, the academic organization of modern universities generally adopts the linear functional organization structure. Management and decision-making does not revolve around the scientific research activity, but takes the administrative unit as the main decision-making body, the academic members of the academic power did not play space. When the academic power of the academic organization members, especial the project leaders is restricted by administrative, scientific research resources will not be allocated reasonably and effectively, which is not conducive to the development of scientific research activities and the optimal combination of scientific research resources.

Therefore, in order to ensure the success of the reform of scientific research organization, we must correctly deal with the division of responsibilities within and between the organization, and balance the autonomy of the organization and the academic freedom of scientific researchers. It is necessary to reform the mode of scientific research organization, and establish the scientific research management system to adapt to the new model. reasonably allocate the functions of teaching and scientific research, so as to realize the redistribution of power. At the same time, it is necessary to solve the problem of power conflict with the traditional organization mode.

\subsection{Open the Discipline Boundary, Innovate the Scientific Research Organization Structure}

The overall optimization level of a system in its internal structure is the one with 
reasonable internal structure. The research system with reasonable internal structure can not only reasonably divide the work between the subsystems of academic organization, but also effectively complement and collaborate. Communication information platform and channel is rich and smooth. The whole system and subsystem of scientific research is coordinated and unified. Therefore, in the process of the reform of scientific research organization in Modern University, the key is to open up the discipline boundary and innovate the organizational structure, and establish a flexible borderless organization matched with collaborative innovation.

The research of Pinch shows that the boundary of discipline and its knowledge production is not fixed and rigid, and the boundaries of knowledge will be redefined in the production of scientific knowledge4. With the development of knowledge, the need for large-scale interdisciplinary research is increasing, and any interdisciplinary cooperation needs must be placed in a certain organizational structure. It is very difficult for the traditional discipline system to meet the interdisciplinary scientific research activities. The flexible borderless organization structure with high flexibility and mobility can promote the integration of disciplines and research personnel, so as to guarantee the innovation resources flow according to certain rules among innovation subjects. However, assemble research team with major research projects or frontier discipline to explore, reform and innovate traditional organization structure, the key is to break through the personnel management system and the operation mode of resource allocation under the traditional discipline system, solve the discipline dilemma of personnel mobility.

With the continuous integration and development of disciplines, each discipline has gradually changed from linear structure to network structure. In order to encourage the researchers of various disciplines to actively participate in the innovative activities of the network structure, the university can form a kind of dynamic scientific research community by making a contract or system to connect them. The research center has gathered academic authority in the field of related disciplines. Management style of the interdisciplinary research organization at the school level completely break the existing organizational boundaries and man-made cognitive disorder, create a new interdisciplinary theoretical system, really promote the open boundaries of the discipline.

\section{Conclusion}

In this paper, the organizational elements, cultural elements, resource elements and other elements of scientific research organizations are discussed, and analyzes the relationship between the above three based on the Theory of Synergy, constructs the theoretical model of modern university scientific research organization, explore collaborative innovation and cooperative development of the key elements such as the goal, structure, personnel, resources, culture and mode of operation of the scientific 
research organization system of modern university around the discipline development. Finally, the paper puts forward the realization pattern of scientific research organization reform: (1)make clear the goal of change, reform the concept of scientific research organization, (2)balance the two type powers, reform the scientific research organization system, (3)open the discipline boundary, innovate the scientific research organization structure.

\section{Acknowledgment}

This work was financially supported by Key Special Funding Project for Hubei Provincial Educational Science and Planning "Research on Innovation of Model of Industry-Academia-Research Collaboration in the Private Universities ” (2015ZA008) fund.

Corresponding author: LIU Chun, Wuhan Technology and Business University, Department of Management, Wuhan 430065, CHINA

Research on Innovation of Model of Industry-Academia-Research Collaboration in the Private Universities

\section{References :}

[1] CHEN Jin. Collaborative innovation [M]. Hangzhou: Zhejiang University press, 2012

[2] LAN Xiao-xia. Research on innovation mechanism of Industry-academia-research collaboration in the United [M]. Beijing: Beijing Jiaotong University press, 2014

[3] ZHOU Shao-nan. The Stanford University [M]. Changsha: Hunan Education Press, 1996

[4] LI Zhong-yun, DENG Xiu-xin. Dilemma, path and policy suggestion of collaborative innovation in universities [J]. China higher education, 2011 (17):11-13.

[5] CHEN Chun-yang. Collaborative innovation is the only way for the development of industry characteristic university [J]. Chinese University Science and technology, 2012 (3):12-19.

[6] LIU Ji-zhen. With the national demand as the goal, the project as the carrier, and actively promote collaborative innovation [J]. Chinese University Science and technology, 2012(3):9-11.

[7] KIM Wei-yin. Study on discipline-crossing theory and crossing-discipline construction in Universities [D]. Suzhou: Soochow University, 2005

[8] Clark, Burton R. Sustaining Change in Universities: Continuities in Case Studies and Concepts[J]. Tertiary Education and Management, 2003, 9(2):99-116.

[9] Trevor Pinch. The Culture of Scientists and Disciplinary [J]. European Journal of Education.1990, 25(3):295-304. 